\title{
Range validation of UWB and Wi-Fi for integrated indoor positioning
}

\author{
Guenther Retscher ${ }^{1}$ (D) Vassilis Gikas ${ }^{2} \cdot$ Hannes Hofer $^{1} \cdot$ Harris Perakis $^{2} \cdot$ Allison Kealy $^{3}$
}

Received: 16 August 2018 / Accepted: 5 December 2018 / Published online: 15 January 2019

(C) The Author(s) 2019

\begin{abstract}
In this paper, we address the challenge of robust indoor positioning using integrated UWB and Wi-Fi measurements. A key limitation of any fusion algorithm is whether the distribution that describes the random errors in the measurements has been correctly specified. Here, we describe the details of a set of practical experiments conducted on a purpose built calibration range, to evaluate the performance of commercial UWB sensors with Wi-Fi measurements as captured by an in-house smartphone application. In this paper, we present comparisons of ranges from the UWB sensors and the Wi-Fi built into the smartphone to true ranges obtained from a robotic total station. This approach is validated in both static and kinematic tests. The calibration range has been established as one component of an indoor laboratory to undertake a more diverse research agenda into robust indoor positioning systems. The experiments presented here have been conducted collaboratively under the joint FIG (WG5.5) and IAG (SC4.2) working groups on multi-sensor systems.
\end{abstract}

Keywords Ultra-wide band (UWB) · Wireless Fidelity (Wi-Fi) · Differential approach · Indoor positioning · Smartphone localization $\cdot$ Performance comparison $\cdot$ System integration

\section{Introduction}

Localization capabilities are nowadays standard features in mobile devices leading to the fact that society has become increasingly reliant on a location-enabled lifestyle (Saeedi and El-Sheimy 2015). Seamless precise navigation in

Guenther Retscher

guenther.retscher@tuwien.ac.at

Vassilis Gikas

vgikas@central.ntua.gr

Hannes Hofer

h.hannes@gmx.at

Harris Perakis

hperakis@central.ntua.gr

Allison Kealy

allison.kealy@rmit.edu.au

1 Department of Geodesy and Geoinformation, TU Wien - Vienna University of Technology, Vienna, Austria

2 School of Rural and Surveying Engineering, National Technical University of Athens (NTUA), Athens, Greece

3 Department of Geospatial Science, RMIT University, Melbourne, VIC, Australia combined outdoor and indoor environments, however, is still a great challenge (see, e.g., (Gikas et al. 2016b; Kealy et al. 2015; Toth et al. 2017)). Application specific requirements of indoor positioning systems (IPS) create the baseline for the performance needs of the solutions under development. Extensive reviews of relevant research have been performed and vary from covering the requirements of navigation and tracking for emergency responders' applications (Fernández and Schön 2017) to pedestrian IPS for mass-market devices (Chen et al. 2015; Correa et al. 2017) and indoor parking modeling (Antoniou et al. 2018; Gikas et al. 2016a). Depending on user requirements and specific case scenario needs, a wide range of localization techniques and technologies is currently available ranging from optical to inertial and radio-based ones. For instance, pedestrian dead reckoning (PDR) exploits the benefits of inertial sensors due to their self-contained functionality (Chen et al. 2015). Similarly, localization problems featuring a group of users employ collaborative positioning techniques while radio-based technologies play a dominant role in the solution provided (Fernandez and Schön 2017; Kealy et al. 2015). In terms of techniques, in addition to traditional approaches, such as angle and range lateration, signal strength/fingerprinting techniques expand rapidly thanks to the advancements in the information technology sector (Gikas and Perakis 2016). Furthermore, new 
computational approaches that make use of artificial intelligence support the development of map-matching and SLAM solutions (Zampella et al. 2015; Zandbergen and Barbeau 2011).

In the last 5 years, the authors of this paper have developed in a number of studies and proposed new concepts for indoor localization, particularly for personal mobility applications (Gikas and Perakis 2016; Hofer and Retscher 2017; Retscher et al. 2017). These investigations have led to a considerable improvement of localization performance in the proposed approaches. In this paper, the concepts developed in previous studies are combined together to even strengthen the navigation solution. To evaluate the achieved performance practical testing was therefore carried out in an indoor lab setting.

The paper is organized as follows: In the "UWB positioning" section, first the characteristics of positioning using ultra-wide band (UWB) are identified followed by brief description of a differential approach for Wi-Fi (Wireless Fidelity) positioning in the "DWi-Fi positioning" section. The "Field-testing in an indoor laboratory" section presents the field test site in a laboratory in the basement of a multistory office building. Then, the "UWB and Wi-Fi integration scenario" section elaborates briefly the integration of UWB and Wi-Fi to strengthen the navigation solution. Finally, brief conclusions are drawn and an outlook on future work is given in the "Conclusions and outlook on future work" section.

\section{UWB positioning}

Using UWB for positioning purposes is a field under rapid development mainly due to the high accuracy and robustness that it provides (Tang et al. 2017; Dewberry and Petroff 2015). Thanks to its high-accuracy ranging potential (Dabove et al. 2018), the basic concept adopted for UWB positioning relies on lateration using ranges calculated based on the Time of Arrival (ToA) technique. Based on RF (radio frequency) signals which spread over a large bandwidth, short pulses transmitted between UWB nodes are utilized for estimating the required travel time for the RF signal. Furthermore, by exploiting the signal characteristics of short pulses, accurate detection of the first pulse (first break) is possible, enabling the range measurement of the direct signal and at the same time filtering out of multipath and NLOS effects (Kim et al. 2018). This functionality is only useful in combination with the ability of the UWB signals to penetrate most construction materialsexcept metal surfaces - and provide accurate ranges. Even though the ToA (Time of Arrival) technique for UWB ranging provides accurate results, exact synchronization (usually through hardware) of the transmitting and receiving devices is a requirement. Through utilizing the coherent transmission capabilities of UWB signals and through implementing the TwoWay Time of Flight (TW-ToF) technique, synchronization issues are resolved to a great extent. TW-ToF relies on the calculation of the time the RF signal requires for traveling from the transmitter to the receiver, the processing and transmission time at the receiver's part and the time for traveling back to the transmitter (see Fig. 1). Furthermore, recent studies (Toth et al. 2017) indicate the potential of UWB technology as it can enhance further the positioning solution through integration with complementary technologies. At implementation stage, Extended Kalman filtering (EKF) (Gibbs 2011; Li et al. 2018) forms the key methodology for computing state estimates for nonlinear case scenarios.

\section{DWi-Fi positioning}

For positioning using Wi-Fi signals, location fingerprinting and (tri)lateration are the most commonly employed techniques (Honkavirta 2008; Chen et al. 2012). They are based on the measurement of the received signal strength (RSS) of the broadcasted Wi-Fi signals. The first technique involves a site survey in a training phase where the RSS to all visible access points (APs) on known reference points (RPs) are scanned and stored in a fingerprinting database. This can cause a very high workload and requires frequent updates of the database. On the other hand, the second technique based on lateration requires the use of theoretical path loss models to convert measured RSS values into ranges between the APs and the user to be able to perform a lateration approach. An empirical derivation of the path loss can be seen as a better strategy. An example for a RSS to range relationship is presented in Fig. 2. In this case, two Raspberry Pi units placed at the two ends of a 50-m-long baseline serve each as
Fig. 1 Illustration of UWB TWToF functionality

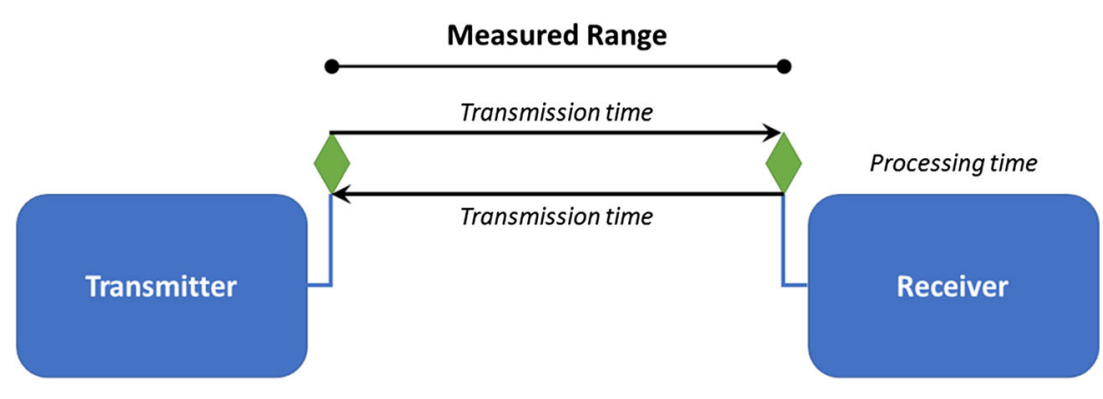


Fig. 2 Example of a RSS to range relationship along a baseline between two Raspberry Pi units serving as APs

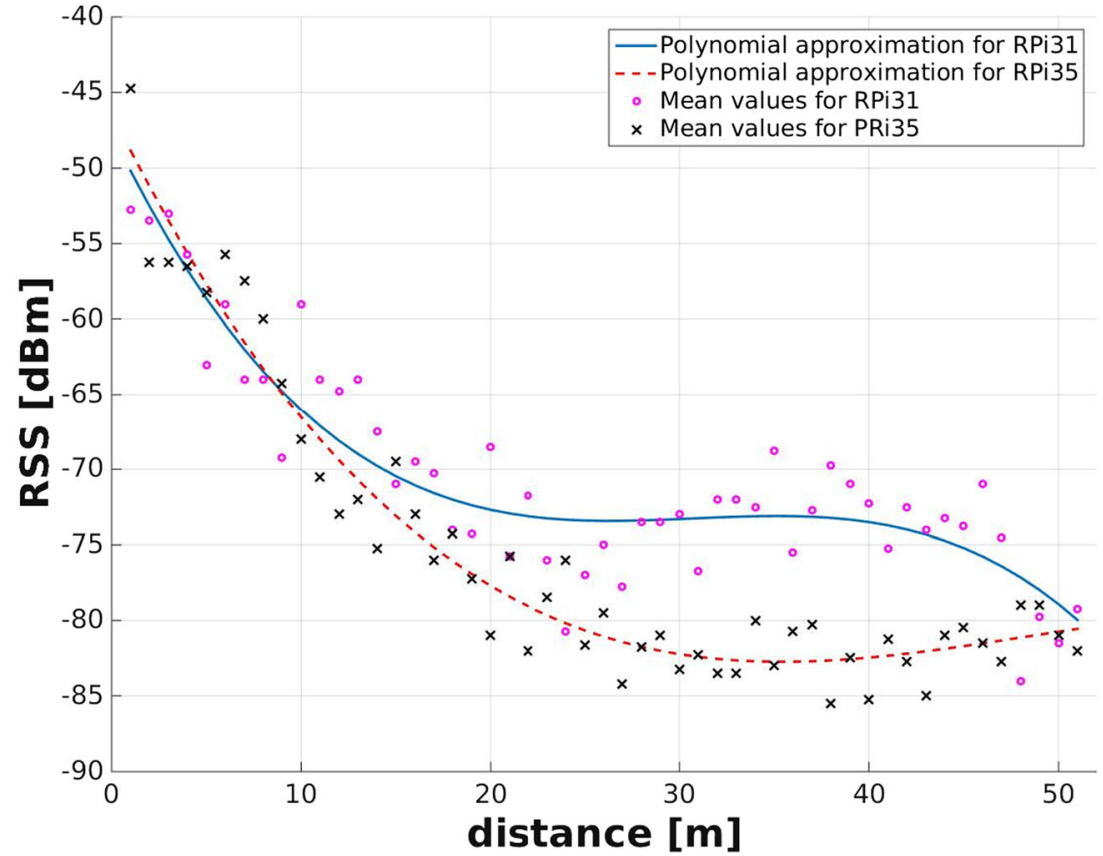

APs. Measurements along the baseline were carried out every meter in four different orientations aligned to the axis of the building. To measure in four orientations is a common praxis in Wi-Fi positioning as then the effect of shielding of an AP with the body if the AP is behind the user can be encountered for. A polynomial approximation was fitted to the curves for the RSS to range relationship. The mean values indicate an average over the four smartphone orientations. As can be seen, the fitted curves are leveling off after around 20 to $25 \mathrm{~m}$. Then, no acceptable relationship between the measured RSS value to the range can be estimated.

Fig. 3 RSS variations plot of observations of five Raspberry Pi units serving as APs

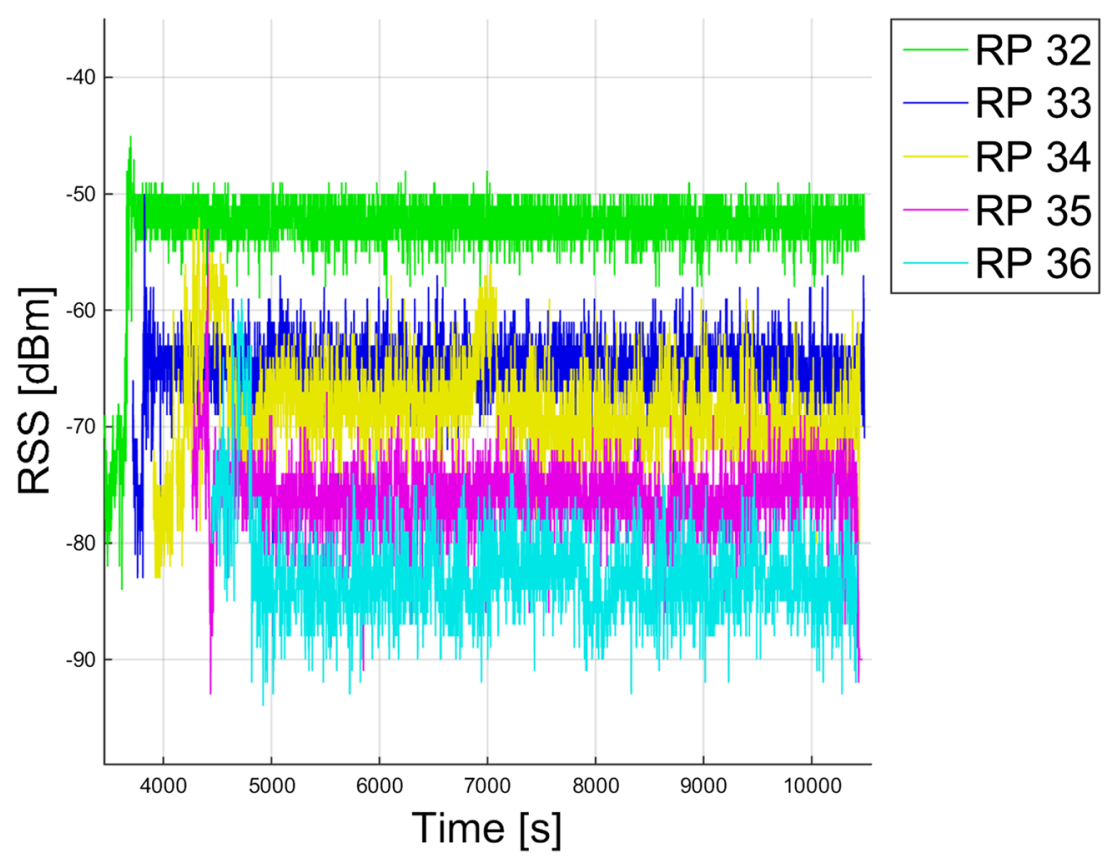

The use of path loss model can be seen as a vulnerability and can cause low positioning accuracies. To improve the RSS to range conversion while considering spatial and temporal Wi-Fi signal variations, a scheme referred to as Differential Wi-Fi (DWi-Fi) is developed by analogy to Differential GPS (DGPS) positioning. The principle of operation is that reference stations (RSs) are placed at known locations in the environment to be able to derive correction parameters. On these RSs, a continuous scanning and monitoring of the RSS values to the other stations-APs and RSs - is then performed. Further information on this differential approach can be found, 
e.g., in (Retscher and Tatschl 2017). Figure 3 shows as an example a record of Wi-Fi RSS values of scans of five Raspberry PI units. As can be seen, the RSS values can be quite noisy indicating high short-time signal fluctuations. Furthermore, other long-time RSS observation revealed that also long-time signal variations can occur, especially if the environments change and also depending on the number of people in the area of interest. Using an area modeling the influence of these variations should be considered when estimating the users' position. Thus, an improvement for the RSS to range conversion is achieved.

In addition to DWi-Fi positioning, (Retscher and Hofer 2017) have developed an approach to include waypoints along the trajectory of the user which are chosen in an intelligent way into the overall navigation solution. This development was referred to as intelligent checkpoint (iCP) concept by the authors. The principle of operation is that waypoints are selected along the users' trajectory under consideration of their dependence following a logical sequence when navigating from a start point $\mathrm{A}$ to a destination $\mathrm{B}$. These iCPs are located on well distinguishable decision points. In the case of indoor positioning, first an entrance has to be chosen when coming from outdoors into a building and then one will enter a foyer or similar area. To reach the next floor, either the stairs or an elevator must be used. Before one can reach the destination room, one has to walk along a corridor from the previous waypoint. Doors, stairways, and corridors can be considered as iCPs which define the possible path. For the identification in indoor environments, a building is then divided into different sections and layers (Hofer and Retscher 2017). The further one enters the building, one reaches a deeper layer of the allocated vector graph. The categories which already describe an easy logical sequence can be derived from these layers. A solution is that all entrances of the building are combined into the first layer. The final layer contains all destination rooms on a certain floor. Then, these layers represent how far one has already progressed inside in a building. If one follows the layer structure from the beginning, only one certain choice of waypoints is always available. To be able to use these logical relationships, however, it is necessary to recognize certain waypoints in a building. With a suitable choice of these iCPs, the structures of the building are usable since in buildings, different bottlenecks must be passed over and over again to reach the destination. These bottlenecks include also structures, such as walls, corners, or doors, which can influence the measured Wi-Fi RSS to the available APs significantly, i.e., much higher or lower values of the RSS can then be measured on different iCPs.

Extending the two concepts described above-DWi-Fi and iCP detection (Retscher et al. 2017) presented the integration of these techniques as well as inertial navigation (IN) using the smartphone sensors (accelerometer, gyroscope, and magnetometer). They could show that an integrated solution yields a satisfactory performance with achievable positioning accuracies of better than $1 \mathrm{~m}$. In the following section, the results of UWB and Wi-Fi positioning are presented followed by a discussion of their integration to strengthen the navigation solution in the "Conclusions and outlook on future work" section.

\section{Field-testing in an indoor laboratory}

In this section, firstly, the test site of the experiments with its setup is presented followed by the achieved results for UWB and Wi-Fi positioning.

\section{Test site}

Experiments were carried out in an indoor lab setting in the second basement of a multi-story office building with a size of approximately $400 \mathrm{~m}^{2}$ (see map in Fig. 4). Five Raspberry Pi units served as reference stations as well as APs. Three additional Wi-Fi routers were deployed in the indoor lab to cover the whole area. For UWB localization, the commercial Time Domain ${ }^{\circledR}$ PulseON OEM (Dewberry and Petroff 2015) was used. Figure 5 shows the Time Domain ${ }^{\circledR}$ PulseON 410 module. The modules use coherent transmission of sequences of short-duration, low duty cycle radio frequency pulses to provide high-quality ranging measurements. Five UWB units

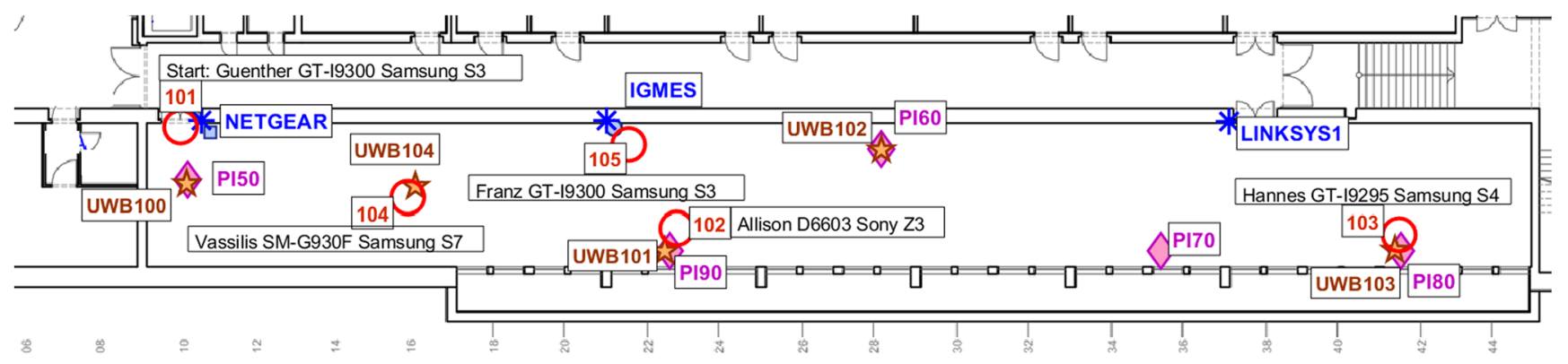

$=10 \mathrm{~m}$

Fig. 4 Location of the sensors in the indoor lab in the second basement of a multi-story office building 


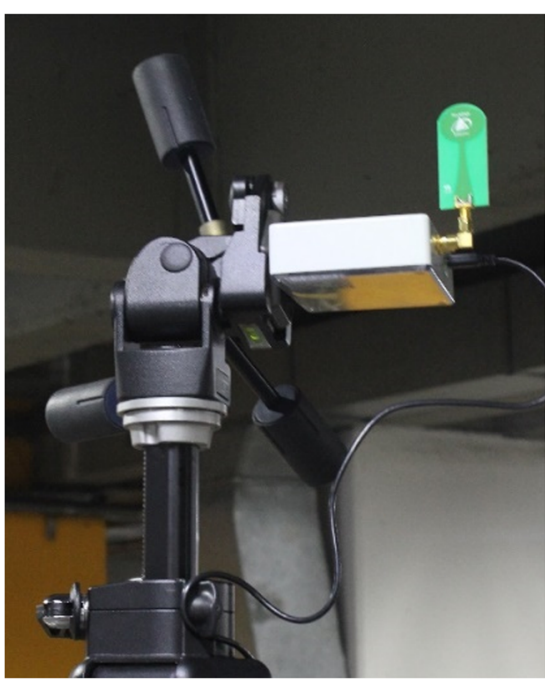

Fig. 5 Time Domain ${ }^{\circledR}$ PulseON 410 module

were used, one (UWB 101) of which was attached to the mobile user and the remaining four (UWB 100, 102, 103, and 104) were collocated with Raspberry Pi units.

Stop-and-go as well as kinematic measurements of five moving users carrying a smartphone each were carried out. Table 1 contains the characteristics of the phones for each user. In the stop-and-go mode, up to 10 RSS scans on each measurement point were carried out. In contrast, a continuous recording of the RSS scans was performed in the kinematic mode.

In the tests presented here, user 1 walked along a trajectory from point 1 to 103 via 104,2 , and 5. The other four smartphone users remained stationary. Ground truth for this experiment was measured using a robotic total station providing position fixes on the $\mathrm{cm}$ accuracy level.

\section{Results of UWB positioning}

Time Domain ${ }^{\circledR}$ PulseON 410 UWB modules generate three types of range measurements using coarse range estimation (CRE) which is based on relative signal strength of the first received pulse, precise range measurement (PRM) which is the outcome of the TW-ToF range estimation and the filtered range estimate (FRE) which is the outcome of an internal Kalman filter implementation of the P 410 module combining the CRE and PRM ranges (Dewberry and Beeler 2012). For

Table 1 Employed smartphones

\begin{tabular}{llcl}
\hline User name & User ID & Start point ID & Smartphone \\
\hline Allison & 2 & 2 & D6603 Sony Z3 \\
Guenther & 1 & 1 & GT-I9300 Samsung S3 \\
Vassilis & 4 & 104 & SM-G930F Samsung S7 \\
Hannes & 3 & 103 & GT-I9295 Samsung S4 \\
Franz & 5 & 5 & GT-I9300 Samsung S3 \\
\hline
\end{tabular}

the validation purposes of the current study, the PRM ranges functionality is selected as the most unaffected outcome of the modules in order to avoid possible filter generated impacts on the FRE range and the instability of the CRE ranges.

Figure 6 presents the time series of the range measurements obtained from the moving UWB node 101 to the four UWB stationary nodes. Cross-comparison of Figs. 4 and 6 reveal the trend in the range observations for all four pairs of nodes. For instance, as expected the ranges observed between nodes UWB 101 and 100 exhibit an increasing pattern, as the mobile node (UWB 101) is moving away from the stationary node (UWB 100), that is, to say the moving user walks from the room entrance (left side of Fig. 4) to the other end of the room. Moreover, the stationary parts acquired during the transition of the mobile user by the Raspberry Pi units correspond to the flat sections. Finally, the high stability in measured ranges is also apparent in the data with the exemption of a small number of epochs (e.g., 200-220 s for units UWB 100-101), which is due to moving or fixed obstacles in the line of sight between the UWB nodes (Retscher et al. 2017).

More specifically, Fig. 7 shows the UWB positioning result of the selected trajectory of user 1 . For this purpose, a fourstate $\left[x, x^{\prime}, y, y^{\prime}\right]$ constant velocity EKF model (Li et al. 2018) was used. The measurement noise for each range measurement is assigned based on the internal error value generated by the P410 UWB module. Filter tuning was applied using a set of empirical values for the driving noise representing the steady gait of the user. In this plot, the stationary parts shown at both ends and at the middle of the trajectory correspond to the flat sections of the range measurements shown in Fig. 6. The positioning solution obtained follows consistently the reference trajectory. However, the deviations observed between the UWB solution and the ground truth obtained using the robotic total station are still large resulting at a mean value $0.76 \mathrm{~m}$ with a standard deviation $0.58 \mathrm{~m}$. This is due to the bad

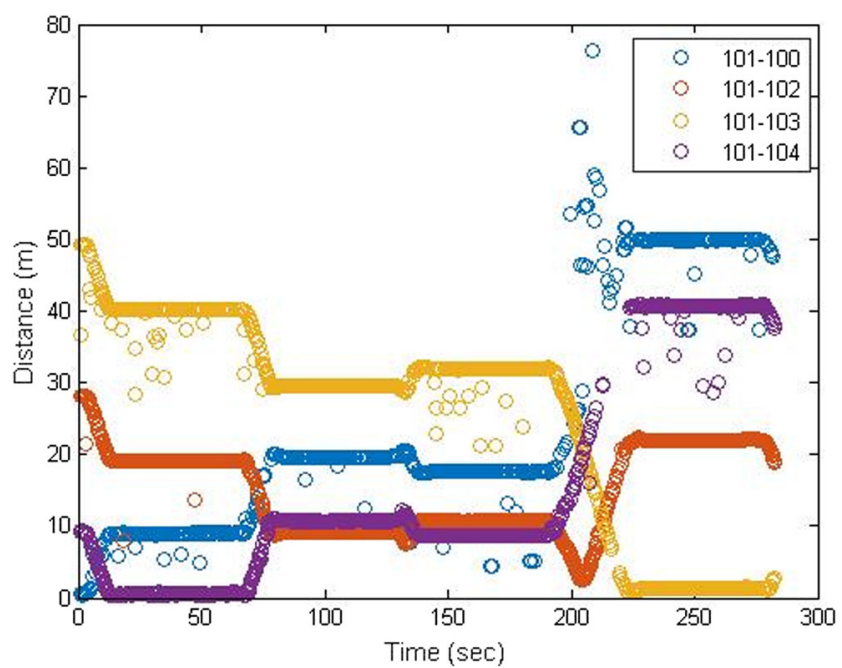

Fig. 6 UWB node 101 range time series derived for all units (source: (Retscher and Tatschl n.d.)) 


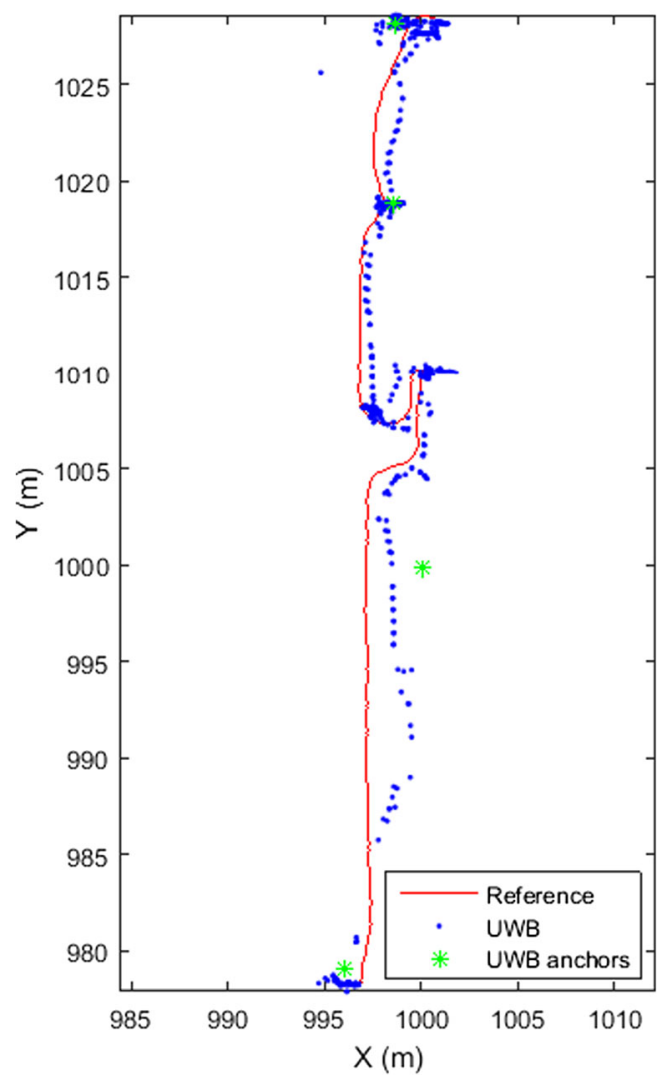

Fig. 7 UWB positioning result

observation geometry between the moving and stationary UWB nodes due to the elongated shape of the lab room. This issue and further limitations of the obtained results are discussed further in the "Limitations of the obtained UWB results" section.

\section{Results of Wi-Fi positioning}

Figure 8 presents a similar representation as in the case of UWB for the Wi-Fi RSS time series of the smartphone users in relation to the Raspberry $\mathrm{Pi}$ unit located at point 103. The total duration is the time which user 1 needed to walk along the trajectory. It is obvious that user 1 walks towards point 103 as the RSS increases significantly from -85 up to $-40 \mathrm{dBm}$. In the case of the other users, the Wi-Fi signals of the Raspberry Pi unit could not always be received and the RSS value drops to the minimum of $-101 \mathrm{dBm}$. Especially, the user 5 could not always measure the RSS values and after two-third of the time not at all. As expected, the user 3 could receive the signals with the highest RSS values in the range of -50 to $-70 \mathrm{dBm}$ throughout the whole time interval. This phone was located next to the PI 80 but the user has shielded the Wi-Fi signals (Retscher et al. 2017).

For the conversion of the RSS into a range, path loss models are required. A suitable model is the so-called one-slope model. It is a very simple empiric model which is based on the principle on the free space loss of the signals. The damping of the signals depends then only on the logarithmic distance between the transmitter and receiver and the reference RSS in the following form:

$P(d)=P_{0}+10 \gamma^{*} \log _{10}(d)$

where $P$ is the received empirical RSS, $P_{0}$ the reference RSS in 1-m distance, $\gamma$ the damping factor, and $d$ the distance between the transmitter and receiver.

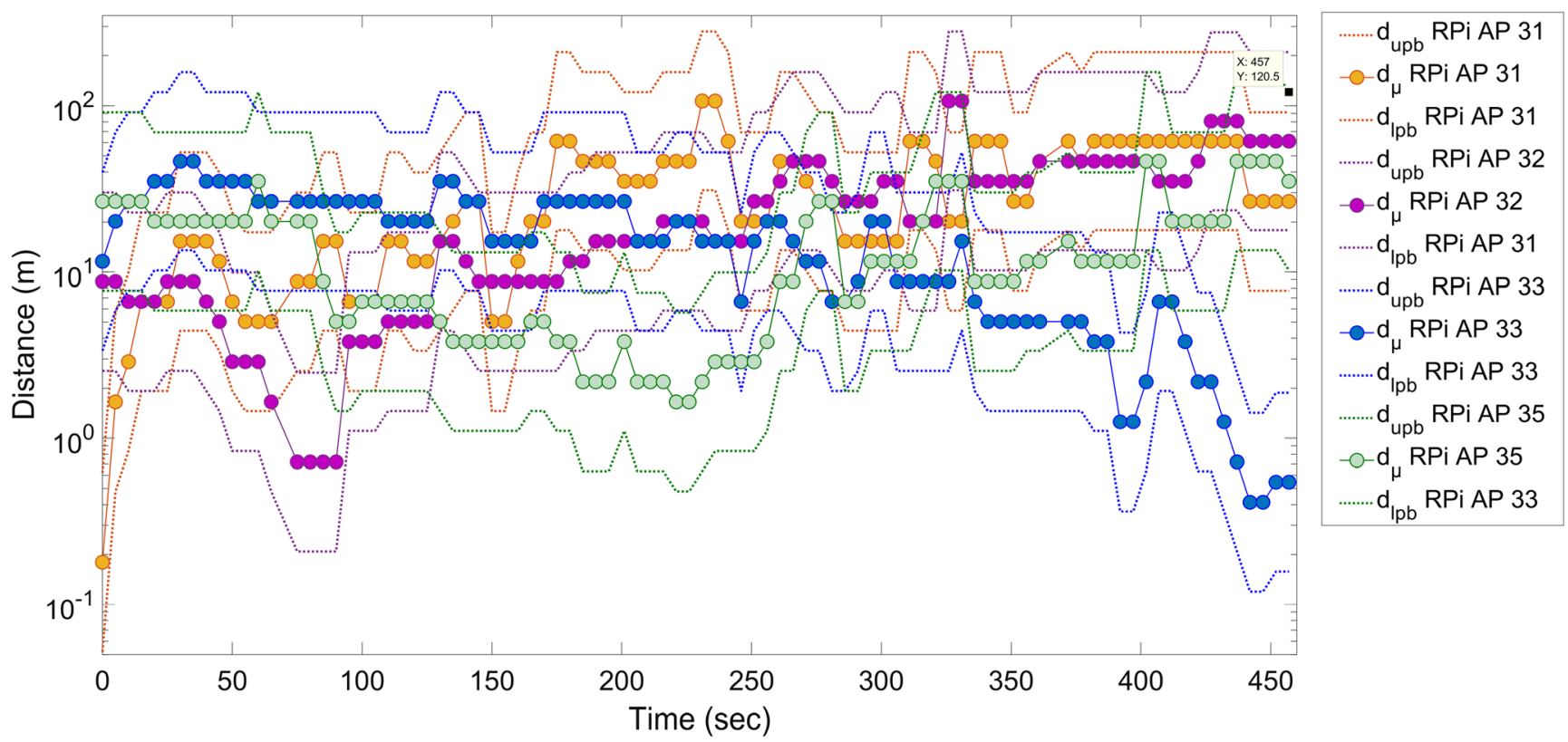

Fig. 8 Wi-Fi RSS range time series derived for all units 


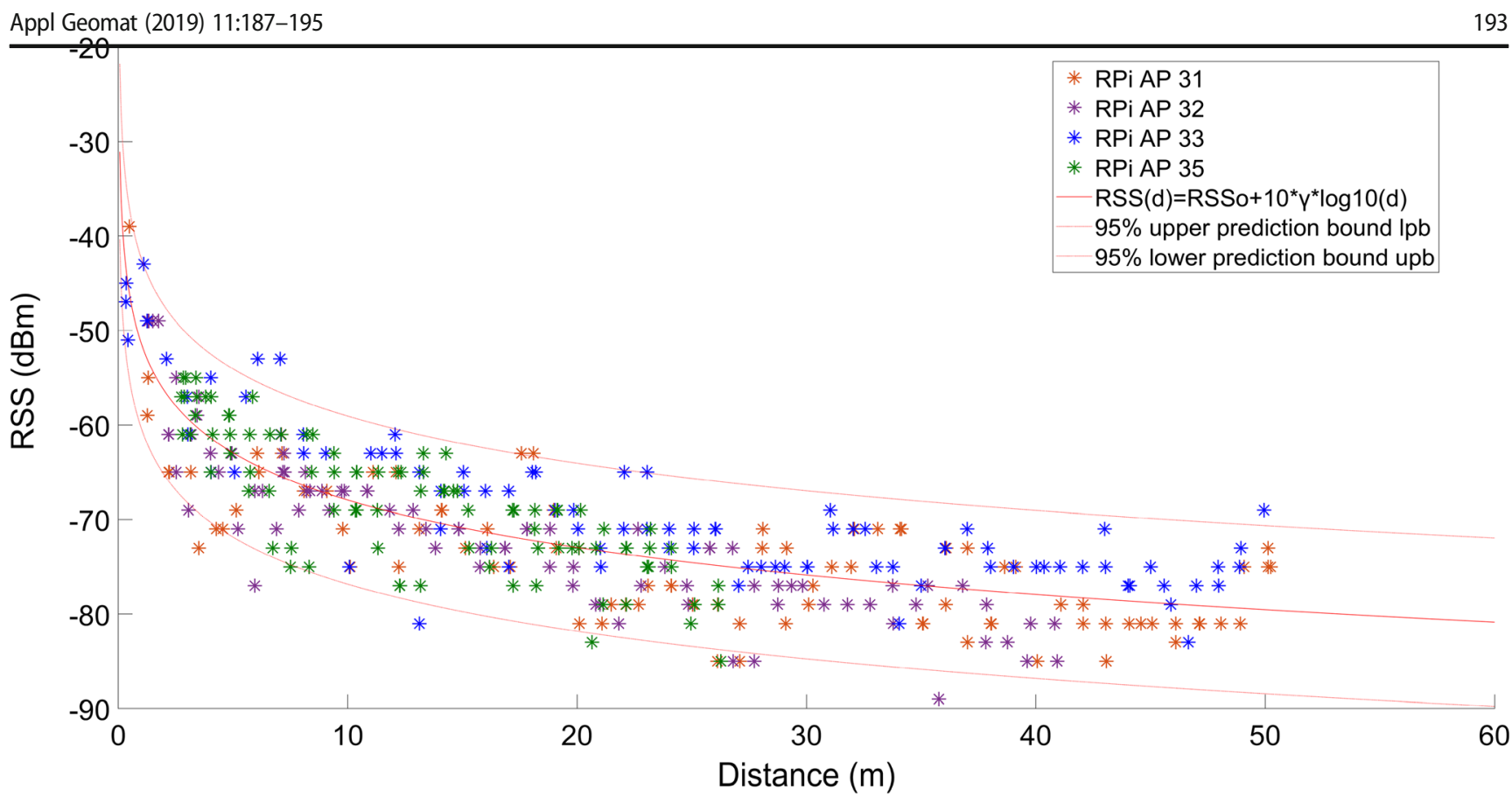

Fig. 9 Range diagram of the one-slope model including upper and lower prediction bound with 95\% reliability level

Figure 9 shows the calculated one-slope model for the measurements. This range diagram describes the relationship between the RSS in $[\mathrm{dBm}]$ and the distance in $[\mathrm{m}]$. The red line in the diagram corresponds to the respected relationship in dependence of the distance from the Raspberry Pi units from the one-slope model. Thereby, $P_{0}$ resulted in a value of $-51.4 \mathrm{dBm}$ and $\gamma$ in -1.66 . The upper and lower prediction bound cover the confidence region for the RSS to range conversion with a reliability level of $95 \%$. For this interval, a range of $\Delta P_{0}$ of $\pm 8.92 \mathrm{dBm}$ is obtained with the same damping factor $\gamma$. Using these values, the observation at the DWi-Fi reference stations RSs can be calibrated. They lead to the resulting range time series presented in Fig. 8. Therefore, the vertical axis has a logarithmic scale.

Finally, Fig. 10 shows the Wi-Fi positioning result of the selected trajectory of user 1 . The mean Euclidean distance from the ground truth measured with the robotic total station resulted in $2.65 \mathrm{~m}$ with a standard deviation of $\pm 1.36 \mathrm{~m}$. The overall minimum deviation obtained is $0.3 \mathrm{~m}$ and the maximum $6.5 \mathrm{~m}$ respectively in all conducted test runs.

\section{Limitations of the obtained UWB results}

Regarding the low-accuracy results of the UWB trajectory along the $X$ axis, the major impact is attributed to the poor geometry of the anchor UWB node positions along the corridor covering in an asymmetrical manner the test area. The long and narrow design of the test area in combination with the requirement for Line-of-Sight (LoS) conditions guided the anchor point selection. However, the current results point to the necessity of evaluating the placement of an additional UWB outside the test area for improving the overall geometry. At the same time, a

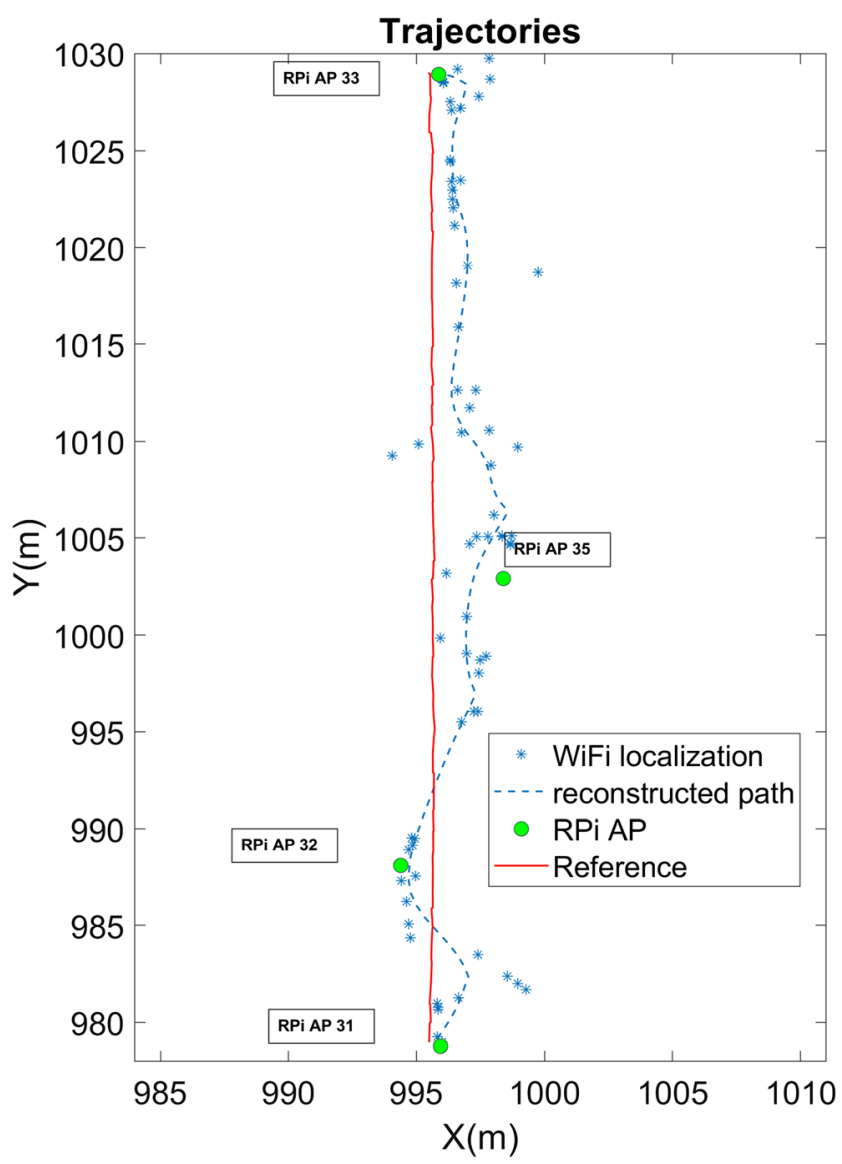

Fig. 10 Wi-Fi positioning result 


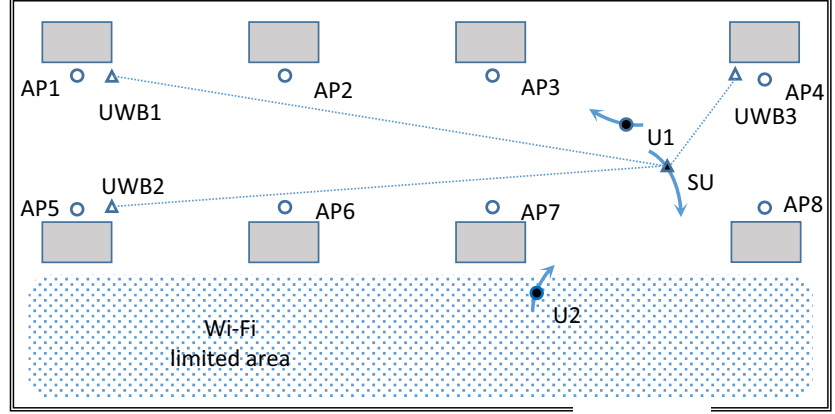

Fig. 11 Combined UWB/Wi-Fi localization scenario

Non-Line-of-Sight (NLOS) ranges weighting technique should take place for mitigating any through-wall signal attenuation effects.

\section{UWB and Wi-Fi integration scenario}

In the experimental work discussed in this study, the role of the UWB system can take two forms depending on the specific goal. Firstly, to provide a high-quality trajectory to serve as a ground truth for the DWi-Fi solution, and secondly to provide further location information (e.g., in the form of control points along a trajectory) for improving the DWi-Fi positioning solution. In this paper, the UWB measurements are used only to produce the range measurements between the moving user and stationary UWB nodes, as a means of quality assurance of the mobile user trajectory. In the future extension, an integration of the UWB and Wi-Fi ranges to the respective anchor nodes and APs is performed for trilateration. Thereby, a meaningful weighting depending on the quality of each range has to be applied. This approach will strengthen the overall navigation solution.

The details for implementing this general approach depends on specific application scenario needs that should accommodate user requirements while preserving cost expenditure. For instance, within an industrial environment tracking effectively assets or goods could be improved if a small number of UWB nodes complement DWi-Fi positioning. In this case, the UWB nodes should be fixed at strategic static locations in the area of interest. Alternatively, UWB nodes could be placed on moving platforms (e.g., key personnel or vehicles) so that they act as dynamic base stations for DWi-Fi users. Figure 11 depicts a localization scenario indoors, in which the super user (SU) that carries also a UWB node, acts as a control point for DWi-Fi users U1 and U2. Obviously, the benefit for user U2 is maximal as he moves in an area with limited Wi-Fi coverage.
Further integration of the UWB/DWi-Fi approach would assume using MEMS IMU information currently available in contemporary smartphones (Gikas et al. 2016b). The in-house developed App (Hofer and Retscher 2017) also records the data of the inertial smartphone sensors. Thus, continuous positioning of the users is enabled augmented by the UWB/DWi-Fi solution serving as absolute localization techniques. This comes along with further challenges, such as adaption of a suitable sensor fusion approach based on an EKF coming along with a meaningful weighting of all observations as well as time synchronization of the sensors with the absolute positioning solution.

Obviously, such a navigation scheme assumes the adoption of near real-time processing tools and thorough quality control procedures to accommodate with the incoming information from disparate data sources. Other applications could benefit from additional sensor types. For example, an approach to large-scale indoor parking facility management would reside on a low-cost RFID Cell-of-Origin ( $\mathrm{CoO})$ solution complemented by UWB/ DWi-Fi (Gikas et al. 2016a; Hofer and Retscher 2017). In this case, the DWi-Fi would guarantee broad coverage while the UWB would increase the overall system accuracy and robustness thanks to its high-accuracy potential and foreseeable decreasing costs.

\section{Conclusions and outlook on future work}

In this paper, the integration of DWi-Fi and detection of waypoints, the so-called iCPs, with UWB range measurements for navigation along the users' trajectory is discussed. In the case of DWi-Fi reference stations (RSs) are deployed where continuous RSS measurements of all visible APs and other RSs are performed. Further information on the operational principle of this approach can be found in (Retscher et al. 2017).

Experiments were conducted in a lab setting. The results in this study demonstrate the performance of UWB and Wi-Fi for range validation. One limitation of the tests, however, was the bad geometry of the location of the UWB anchor nodes as the lab has a length over nearly $60 \mathrm{~m}$ and a width of only around $5 \mathrm{~m}$. Also in the case of Wi-Fi positioning, the intersection for trilateration from the ranges to the Raspberry Pi units was not optimal.

Further testing in combined out-/indoor environments was conducted in a new measurement campaign at the Ohio State University, USA, in the first week of October 2017. This work is carried out from the joint FIG (WG5.5) and IAG (SC4.2) working groups on multisensor systems. Currently, the described approaches in this paper are applied to these measurements. 
Acknowledgments The authors would like to thank Mr. Franz Obex for participating in the data collection and his ideas and cooperation and $\mathrm{Mr}$. Jonathan Kleine and Mrs. Lisa Whitemore for the preparation of Figs. 2 and 3 respectively.

Funding Information Open access funding provided by TU Wien (TUW).

Open Access This article is distributed under the terms of the Creative Commons Attribution 4.0 International License (http:// creativecommons.org/licenses/by/4.0/), which permits unrestricted use, distribution, and reproduction in any medium, provided you give appropriate credit to the original author(s) and the source, provide a link to the Creative Commons license, and indicate if changes were made.

\section{References}

Antoniou C, Gikas V, Papathanasopoulou V, Mpimis T, Perakis H, Kyriazis C (2018) A framework for risk reduction for indoor parking facilities under constraints using positioning technologies. Int $\mathrm{J}$ Disaster Risk Reduct 31:1166-1176

Chen R, Pei L, Liu J, Leppäkoski H (2012) WLAN and Bluetooth positioning in smart phones. In: Chen R (ed) Ubiquitous positioning and mobile location-based services in smart phones. Hershey PA. IGI Global, USA, pp 44-68

Chen G, Meng X, Wang Y, Zhang Y, Tian P, Yang H (2015) Integrated WiFi/PDR/smartphone using an unscented Kalman filter algorithm for 3D indoor localization. Sensors 15(9):24595-24614

Correa A, Barcelo M, Morell A, Vicario JL (2017) A review of pedestrian indoor positioning systems for mass market applications. Sensors 17(8): 1927

Dabove P, Di Pietra V, Piras M, Jabbar AA, Kazim SA (2018) Indoor positioning using ultra-wide band (UWB) technologies: positioning accuracies and sensors' performances. IEEE/ION Position, Location and Navigation Symposium (PLANS), 175-184

Dewberry B, Beeler W (2012) Increased ranging capacity using Ultrawideband direct-path pulse signal strength with dynamic recalibration. IEEE/ION Position, Location and Navigation Symposium (PLANS), 23-26 April 2012

Dewberry B, Petroff A (2015) Precison navigation with AD-HOC Autosurvey using ultrawideband two-way ranging network. 12th IEEE Workshop on Positioning, Navigation and Communication WPNC'15, Dresden, Germany, March 11-12, 2015, 6 pgs

Fernández NZ, Schön S (2017) Development of a simulation tool for collaborative navigation systems. 14th Workshop on Positioning, Navigation and Communications (WPNC), Bremen, pp. 1-6

Gibbs BP (2011) Advanced Kalman filtering, least-squares and modeling: a practical handbook. Willey, ISBN: 978-0-470-52970-6, p. 632
Gikas V, Perakis H (2016) Rigorous performance evaluation of smartphone GNSS/IMU sensors for ITS applications. Sensors 16(8):1240 1-21

Gikas V, Antoniou C, Retscher G, Panagopoulos AD, Perakis H, Kealy A, Mpimis T (2016a) A low-cost wireless sensors positioning solution for indoor parking facilities management. Journal of Location Based Services 10(3):1-21

Gikas V, Retscher G, Kealy A, Zhang K, Paffenholz J-A, Ruotsalainen L, Perakis H, Santos M (2016b) IAG SC 4.1 emerging positioning technologies and applications: objectives and structure for the term 2015-19. European Navigation Conference, Helsinki, Finland, May 30 - Jun 02, 2016

Hofer H, Retscher G (2017) Seamless navigation using GNSS and Wi-Fi/ IN with intelligent check-points. Journal of Location Based Services 11(3-4):204-221

Honkavirta V (2008) Location fingerprinting methods in wireless local area networks. Master of Science Thesis, Tampere University of Technology, Finland

Kealy A, Retscher G, Toth C, Hasnur-Rabiain A, Gikas V, GrejnerBrzezinska D, Danezis C, Moore T (2015) Collaborative navigation as a solution for PNT applications in GNSS challenged environments - report on field trials of a joint FIG/IAG working group. J Appl Geodesy 9(4):244-263

Kim DH, Kwon GR, Pyun JY, Kim JW (2018) NLOS Identification in UWB Channel for Indoor Positioning. 15th IEEE Annual Consumer Communications and Networking Conference (CCNC), 1-4

Li X, Wang Y, Liu D (2018) Research on extended Kalman filter and particle filter combinational algorithm in UWB and foot-mounted IMU fusion positioning. Mob Inf Syst 2018:1-17

Retscher G, Hofer H (2017) Wi-Fi location fingerprinting using an intelligent checkpoint sequence. J Appl Geodesy 11(3):197-205

Retscher G, Tatschl T (2017) Indoor positioning with differential Wi-Fi Lateration. J Appl Geodesy 11(4):249-269

Retscher G, Hofer H, Kealy A, Gikas V, Obex F (2017) Cooperative localization in indoor environments using constrained differential Wi-Fi and UWB measurements. ION GNSS++ Conference 2017, Institute of Navigation: Portland, Oregon, USA

Saeedi S, El-Sheimy N (2015) Activity recognition using fusion of lowcost sensors on a smartphone for mobile navigation application. Micromachines 6(8):1100-1134

Tang Y, Wang J, Li C (2017) Short-range indoor localization using a hybrid Doppler-UWB system. IEEE MTT-S International Microwave Symposium (IMS), 1011-1014

Toth CK, Jozkow G, Koppanyi Z, Grejner-Brzezinska D (2017) Positioning slow-moving platforms by UWB technology in GPSchallenged areas. J Surv Eng 143(4):04017011

Zampella F, Jiménez A, Seco A, Fernando (2015) Indoor positioning using efficient map matching, RSS measurements, and an improved motion model. IEEE Trans Veh Technol 64:1. https://doi.org/10. 1109/TVT.2015.2391296

Zandbergen PA, Barbeau SJ (2011) Positional accuracy of assisted GPS data from high-sensitivity GPS-enabled mobile phones. J Navig 64(3):381-399 